\title{
Small Volumes in Biology
}

Daniel Axelrod ${ }^{1 *}$, Jeremy J. Axelrod ${ }^{2}$, Ronald W. Holz ${ }^{3}$, Nancy L. Thompson ${ }^{4}$

1. Dept. of Physics, and LSA Biophysics, University of Michigan, Ann Arbor, MI, USA.

${ }^{2}$. Dept. of Physics, University of California, Berkeley, Berkeley, CA, USA.

3 . Dept. of Pharmacology, University of Michigan, Ann Arbor, MI USA.

4. Dept. of Chemistry, University of North Carolina, Chapel Hill, NC, USA.

* Corresponding author: daxelrod@umich.edu

Biological cells are small compartments that themselves often contain subcompartments smaller than the optical resolution limit of a microscope. We discuss here some examples of the ways that submicroscopic small biological compartments (such as the nucleus, secretory granules, and endocytotic vesicles) can be studied by specialized optics, how they themselves can affect optical properties, and how the smallness of the volume can affect chemical kinetic rates inside the compartments.

One such specialized technique is total internal reflection fluorescence (TIRF), along with its combinations with other techniques. TIRF techniques depend on collimated light incident upon an interface at greater than some critical angle between a substrate (usually a glass coverslip) and a lower refractive index medium above, usually a clear liquid compatible with living cells, thereby forming an exponentially-decaying evanescent field in the lower index side of the interface.

With an emphasis on the optical systems, we will briefly review the combination of total internal reflection (TIR) excitation with fluorescence recovery after photobleaching (TIR-FRAP) and with polarization (pTIRF), and describe their application to the study of secretory granules (Fig. 1). TIR-FRAP can measure the diffusion rate of fluorescence-marked molecules inside or on the surface of a tiny secretory granule [1]; pTIRF can detect the deformation of a plasma membrane as a secretory granule fuses with it, and detect whether the deformation precedes or persists after the fusion event [2].

A clean evanescent field depends upon the interface being smooth. But the objects under study - the cells and its subcellular compartments - themselves disrupt the evanescent field because these objects have a spatially variable refractive index. It is important for the interpretability (and even the viability) of the TIR technique that optical scattering does not severely degrade the thin exponential profile of the evanescent field and thereby lead to unwanted illumination of objects beyond. We present a first-order theoretical approximation, starting with Maxwell's Equations, to calculate the intensity and propagation directions of scattered light for any general pattern of refractive index variation (Fig. 2). From this, we can predict theoretically that the scattered light intensity typically will be $<5 \%$ of the incident evanescent field intensity for the refractive index variations commonly found in cell culture monolayer samples. The pattern and intensity of the scattered light depends on the polarization of the incident light and on the size and morphology of the refractive index variations [3].

An interesting set of theoretical questions arises because of the ubiquitous presence of small compartments in biology. How do small compartments affect the extent, likelihood, and speed of chemical reactions (in particular, the rate of relaxation from a disturbance away from equilibrium) within them? We have carried out calculations with reaction-diffusion theory to determine that small volumes tend to substantially increase the rate of chemical reactions (over open volume rates) between a diffusing reactant confined within a small volume and an immobile target in the center of the volume (Fig. 3) [4,5]. The physical reasons and implications for this enhancement will be discussed [6]. 


\section{References:}

[1] A Ngatchou-Weiss, MA Bittner, RW Holz and D Axelrod, Biophys. J. 107 (2014), p.16.

[2] A Anantharam, B Onoa, RH Edwards, RW Holz, and D Axelrod, J. Cell Biol. 188 (2010), p. 415.

[3] J Axelrod and D Axelrod, Biophys. J. 114 (2018), p. 533A.

[4] NL Thompson and TP Burghardt, Biophys. Chem. 21 (1985), p. 173.

[5] NL Thompson and D Axelrod, in preparation (2019).

[6] The authors acknowledge funding: NSF DGE 1752814 ( JJA); NIH R01-170553 (RWH and DA).

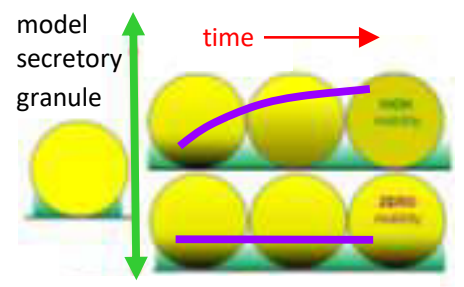

TIR bleach TIR probe
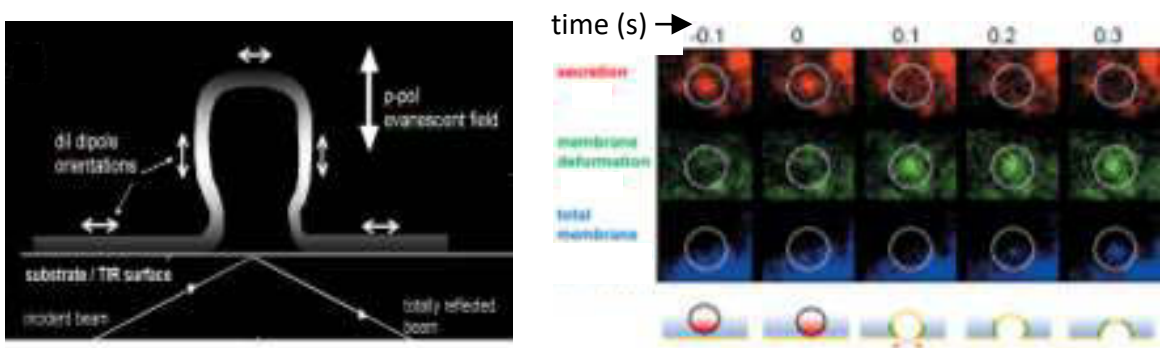

Figure 1. (Left) TIR-FRAP scheme on secretory granule with high or low mobility contents. Purple lines represent post-bleach fluorescence vs. time. (Middle) P-FRAP scheme on diI-labeled membrane with brightest fluorescence at regions non-parallel with the substrate coverslip. (Right) Actual secretion event in chromaffin cell; top shows fluorescence material in granule lost upon fusion with membrane; middle shows polarized diI fluorescence ratio which highlights a long-lasting deformation; bottom shows total membrane fluorescence. Strip along bottom shows putative sequence of fusion structures.
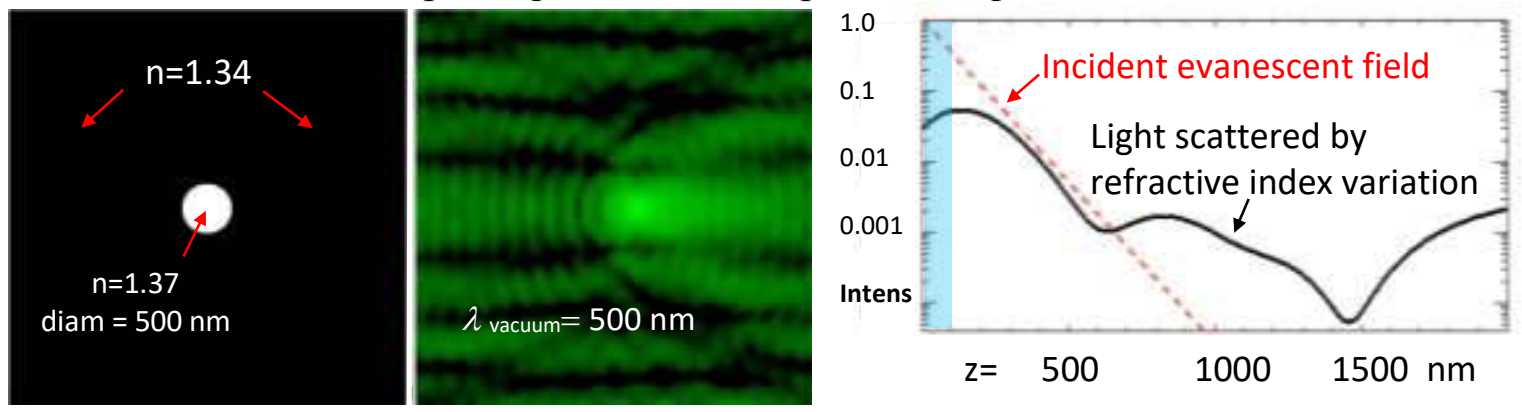

Figure 2. Theoretical scattering of an evanescent field (traveling left-to-right) by a subcellular component (Left) modeled by a circular refractive index variation in $\mathrm{x}-\mathrm{y}$ plane with a $\mathrm{z}$-height much greater than the evanescent field depth. (Middle) Consequent scattered intensity at $\mathrm{z}=0$ shown on a $\log$ scale to highlight dimmer detail; actual intensity is more contrasty. (Right) z-dependence of scattered intensity at brightest $\mathrm{x}-\mathrm{y}$ location compared to incident evanescent intensity of characteristic depth $130 \mathrm{~nm}$, normalized to incident intensity $=1.0$ at $\mathrm{z}=0$. Scattered intensity is less than $10 \%$ of the incident intensity only in the first hundred $\mathrm{nm}$ of $\mathrm{z}$, shown in blue shading.
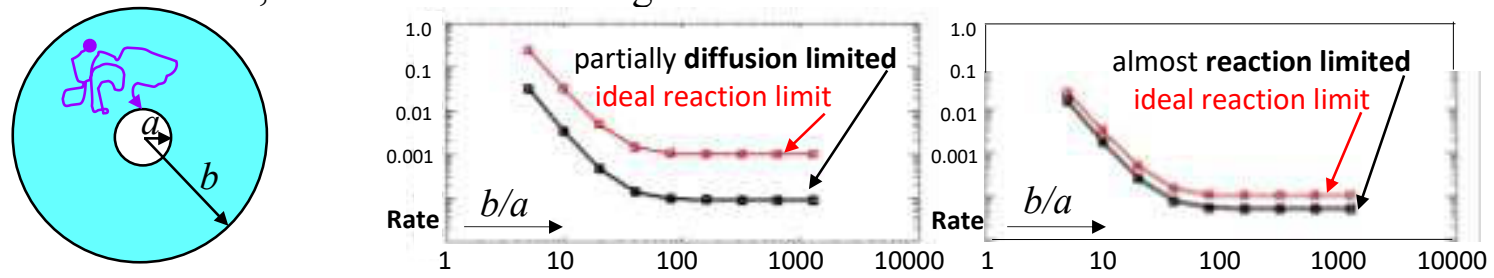

Figure 3. (Left) A model of reactant binding to a target of radius $a$ at the center of an impermeant sphere of radius $b$. (Middle and Right) Relaxation rates vs. the ratio $b / a$. Black curves are the calculated rates including the slowing effect of diffusion; red curves are "reaction limit" rates in which diffusion is fast. In all cases, reaction rates with the target are several orders of magnitude faster for small volumes. Two different sets of rates are shown, either partially "diffusion limited" or almost "reaction limited". 